\title{
Forest Height Extraction from PolInSAR Image Using a Hybrid Method
}

\author{
Nghia Pham Minh ${ }^{1}$, Chengyi Wang ${ }^{2 *}$, Bin Zou ${ }^{1}$, \\ Quang Thi Nguyen ${ }^{3}$ and Van Nhu Le ${ }^{4}$ \\ ${ }^{1}$ Dept. of Information Engineering, Harbin Institute of Technology, Harbin, China \\ ${ }^{2}$ Forestry Research Institute of Heilongjang Province, Harbin, China \\ ${ }^{3}$ School of Electronics and Information engineering, Changchun University of Science \\ and Technology \\ ${ }^{4}$ Research center for Space Optics Engineering, Harbin Institute of Technology, \\ China \\ *Corresponding author: Chengyi Wang, Email: wcy758@163.com
}

\begin{abstract}
Forest height is one of the most important vegetation vertical structure for many forest management activities. It is closely related with forest biomass and absorption of carbon. PolInSAR is a promising remote sensing technique that has been frequently used for extracting forest height. Recently, there have been plenty of research on the retrieval of vegetation parameters by single frequency single baseline PolInSAR such as the ESPRIT method, three-stage inversion. However, these methods tend to underestimate the forest height due to attenuations of the electromagnetic waves in the ground medium. This paper proposes a method to improve the accuracy of forest height estimation. This method is based on a fusion of hybrid method extracting forest parameters and the coherence amplitude method. The proposed algorithm performance is evaluated with simulated data from PolSARProSim software and ALOS PALSAR L-band polarimetric interferometric SAR data.
\end{abstract}

Keywords: PolInSAR, ESPRIT, forest height estimation, coherence amplitude method

\section{Introduction}

Forest are tree dominated plant formations that cover approximate $30 \%$ of the total land area [1], with a mean tree height of about $20 \mathrm{~m}$. Forest height is important information for many management activities and is a critical parameter in modeling of ecosystem procedures $[16,17]$. Polarimetric interferometric synthetic aperture radar (PolInSAR) system have shown a great potential for forest height retrieval as it is sensitive to the vertical structure and physical characteristics of the scattering media. The inversion approaches are based on the sensitivity of the radar interferometric measurement (phase and coherence) to the vertical distribution of scattering elements (leaves, branches, and trunks), combined to the sensitivity of radar polarimetry to shape and orientation of these elements. Polarimetric interferometry, which is interferometry between all possible polarizations, similar at each end of the baseline, is therefore sensitive to 3-D distribution of scatterers. Interferograms obtained at different polarizations correspond then to different scattering centers located at different heights in the canopy depending on each ground-to-canopy power ratio [18].

Two decades ago, several techniques have been proposed for forest height estimation using single baseline PolInSAR image such as ESPRIT method [2-4], three-stage inversion [5], and 
algorithms based on the Maximum Likelihood Estimation [6, 7]. The three-stage inversion algorithm proposed by S. R. Cloude and K. P. Papathanassiou is quite simple and most widely used. However, in the three-stage inversion algorithm, the estimation of volume decorrelation is not very accurate and there is an ambiguity zone of volume decorrelation. On other hand, the three-stage algorithm requires multiple parameter least-square estimation which is complex and often becomes ill-conditioned. The ESPRIT technique can detect local scattering centers corresponding to the canopy top and ground in the forest area but detection accuracy of the technique becomes worse for dense forest regions due to strong volume scattering component. For that reason, we propose a hybrid method for forest height estimation from PolInSAR image.

The aim of this paper to present an accuracy improvement of forest height estimation by using the hybrid method. The proposed method is combination of two canonical forest height estimation approaches for PolInSAR data. Firstly, the ground topographic phase is estimated by the coherence line method while the effect phase center of forest canopy is obtained using ESPRIT algorithm. However, in order to improve accuracy for canopy phase estimation we implement Freeman and Durden decomposition technique [8] to detect the volume scattering before applying the ESPRIT. Secondly, the forest height is estimated by phase differencing. Thirdly, the height is compensated by the coherence amplitude algorithm. The propose method enable not only of improve the accuracy of forest height estimation but also classification between forest areas and other area such as urban, vegetation, road etc. Experimental results show that accuracy of the forest height can be improved by this scheme.

The organization of this paper is as follows. In section 2, we present underlying ground topography phase estimation and ESPRIT algorithm. The retrieval of forest height from the proposed algorithm is delivered in section 3. The experimental results of the parametric inversion with simulated data and spaceborne data are presented and discussed in section 4. Finally, the conclusion and future work are drawn in section 5.

\section{Ground Phase Estimated using TLS Line Fit Method and ESPRIT Algorithm}

\subsection{Scattering Model}

A monostatic, fully polarimetric interferometric SAR system are measured for each resolution cell in the scene from two slightly different look angles, two scattering matrices $\left[S_{1}\right]$ and $\left[S_{2}\right]$. In the case of backscattering in a reciprocal medium, the individual polarimetric data sets may be expressed by means of the Pauli target vector [9].

$$
\vec{k}_{i}=\frac{1}{\sqrt{2}}\left[\begin{array}{lll}
S_{h h}^{i}+S_{v v}^{i} & S_{h h}^{i}-S_{v v}^{i} & 2 S_{h v}^{i}
\end{array}\right]^{T}
$$

where ${ }^{T}$ represents the vector transposition, $S_{p q}(p, q=\{h, v\})$ are the complex scattering coefficients and $i=1,2$ denote measurements at two ends of the baseline.

The basic radar observable in PolInSAR is a six-dimension complex matrix of a pixel in each resolution element in the scene, defined as shown in (2)

$$
[T]=\left\langle\vec{k} \cdot \vec{k}{ }^{*} T\right\rangle=\left\lceil\begin{array}{cc}
T_{1} & \Omega \\
\Omega{ }^{*} T & T_{2}
\end{array}\right] \quad \text { with } \quad \vec{k}=\left[\begin{array}{l}
\overrightarrow{k_{1}} \\
\overrightarrow{k_{2}}
\end{array}\right]
$$


where $\langle\bullet\rangle$ denotes the ensemble average in the data processing and " represents the complex conjugation. $\left[T_{1}\right]$ and $\left[T_{2}\right]$ are the conventional Hermitian polarimetric coherence matrices which describe the polarimetric properties for each individual image separately, while $[\Omega]$ is a non-Hermitian complex matrix which contains polarimetric and interferometric information.

In general, the complex polarimetric interferometric coherence as a function of the polarization of the two images is given by

$$
\tilde{\gamma}\left(\vec{\omega}_{1}, \vec{\omega}_{2}\right)=\frac{\vec{\omega}_{1}^{* T} \Omega \vec{\omega}_{2}}{\sqrt{\left(\vec{\omega}_{1}^{*} T_{1} \vec{\omega}_{1}\right)\left(\vec{\omega}_{2}{ }^{*} T_{2} \vec{\omega}_{2}\right)}}=\frac{\vec{\omega}{ }^{* T} \Omega \vec{\omega}}{\vec{\omega}^{* T} T \vec{\omega}}
$$

where $\vec{\omega}_{1}=\vec{\omega}_{2}=\vec{\omega}$ is a three-component unitary complex vector defining the selection of each polarization stage, and $T=\left(T_{1}+T_{2}\right) / 2$.

In the forest observation at L-band, both ground and canopy back scattering are contained in the received signals. A most simple model to describe such as a scenario the random volume over ground $(\mathrm{RVoG})$ scattering model $[5,9]$. This model can be described as:

$$
\begin{aligned}
& \tilde{\gamma}(\vec{\omega})=e^{j \phi_{0}} \frac{\tilde{\gamma}_{v}+m(\vec{\omega})}{1+m(\vec{\omega})}=e^{j \phi_{0}}\left(\tilde{\gamma}_{v}+\frac{m(\vec{\omega})}{1+m(\vec{\omega})}\left(1-\tilde{\gamma}_{v}\right)\right) \\
& =e^{j \phi_{0}}\left(\tilde{\gamma}_{v}+L(\vec{\omega})\left(1-\tilde{\gamma}_{v}\right)\right) \quad \text { with } 0 \leq L(\vec{\omega}) \leq 1
\end{aligned}
$$

where $\phi_{0}$ is the phase related to the ground topography and $m(\vec{\omega})$ represents the effective ground-to-volume amplitude ratio accounting for the attenuation through the volume. The vector $\vec{\omega}$ is a unit complex vector defining the choice of polarization, and $\tilde{\gamma}_{v}$ denotes the complex coherence for the volume alone, which depends on the mean wave extinction and forest height as introduced in $[5,10,11]$.

\subsection{Underlying Ground Topography Phase Estimation using Total Least Squares Line Fit Model}

Unlike previous line fit techniques, that employ only two coherence values, selected either on the basic of scattering physics or by employing coherence optimization [11]. In this paper, we use a more robust version of these approaches to employ multiple polarization channels and apply a least squares line fit method to the multiple complex data points. In this way, we can avoid problem of any selected pair of points becoming too close, and thus minimize error in the surface topography estimation.

In this method, we use the linear coherence loci assumption to generate a linear relationship between the real and imaginary parts of the complex coherence, as show in Figure 1. In order to reduces error for estimation of two coefficients $M$ and $C$ of line fit, we employ a total least squares solution (TLS) that account for errors in both $x$ and $y$. Geometrically, the TLS approach amounts to using a different measure of distance: the perpendicular distance $R_{i}$, at right in Fig. 1 and related to $\Delta y$ as shown in Eq. (5) 


$$
\begin{aligned}
R_{i} & =(\Delta y) \cos (\theta)=\frac{\Delta y}{\sqrt{1+\tan ^{2}(\theta)}} \\
& =\frac{\Delta y}{\sqrt{1+M^{2}}}
\end{aligned}
$$

Then, the function to be minimized has the following form:

$$
\sum_{i=1}^{N} R_{i}=\frac{1}{1+M^{2}} \sum_{i=1}^{N}\left(y_{i}-C-M x_{i}\right)^{2}
$$

Through taking the differentiate the left side of Eq. (6) with respect to $\mathrm{M}$ and $\mathrm{C}$, we can obtain a direct solution for the estimate of $\mathrm{C}$ and $\mathrm{M}$ as follows

$$
\begin{aligned}
& C=\frac{1}{N}\left(\sum_{i=1}^{N} y_{i}-M \sum_{i=1}^{N} x_{i}\right)=\bar{y}-M \bar{x} \\
& M=\frac{-C_{1} \pm \sqrt{C_{1}^{2}-4 C_{0} C_{2}}}{2 C_{2}}
\end{aligned}
$$

where the three coefficient are defined in terms of the data points as follows:

$$
\begin{gathered}
C_{0}=-\sum_{i=1}^{N}\left(y_{i}-\bar{y}\right)\left(x_{i}-\bar{x}\right) \\
C_{1}=\sum_{i=1}^{N}\left\{\left(x_{i}-\bar{x}\right)^{2}-\left(y_{i}-\bar{y}\right)^{2}\right\} \\
C_{2}=\sum_{i=1}^{N}\left(y_{i}-\bar{y}\right)\left(x_{i}-\bar{x}\right) \\
\qquad \begin{array}{l}
\operatorname{Im}(\tilde{\gamma}) \boldsymbol{I m}(\tilde{\gamma})=M \operatorname{Re}(\tilde{\gamma})+C \\
y=M x+C
\end{array}
\end{gathered}
$$

Figure 1. Geometric of Total Least Squares Line Fit Approach

This then provides us with a method for fitting a line to an arbitrary number of polarization channels. Finally, we can use the estimate of the two coefficient $M$ and $C$ to find the two unit circle intersection points as show in Figure 2. These two points can be found explicitly in terms of $M$ and $C$ which is given by 


$$
\left.\left.\begin{array}{l}
x^{2}+y^{2}=1 \\
y=M x+C
\end{array}\right\} \Rightarrow \begin{array}{l}
x_{P}=\frac{-M C \pm \sqrt{M^{2}-C^{2}+1}}{1+M^{2}} \\
y_{P}=M x_{P}+C
\end{array}\right\} \Rightarrow e^{j \phi}=x_{P}+j y_{P}
$$

The TLS approach employs $\mathrm{N}=12$ typical polarizations, such as $\mathrm{HH}, \mathrm{VV}, \mathrm{HV}, \mathrm{HH}-\mathrm{VV}$, $\mathrm{HH}+\mathrm{VV}, \tilde{\gamma}_{i}^{o p t} \ldots$ to do the total least squares line fit for the extraction of the line segment mentioned in the forest model. This approach is often combined with the coherence separation optimization method to decide which phase point to use as the topography estimate.

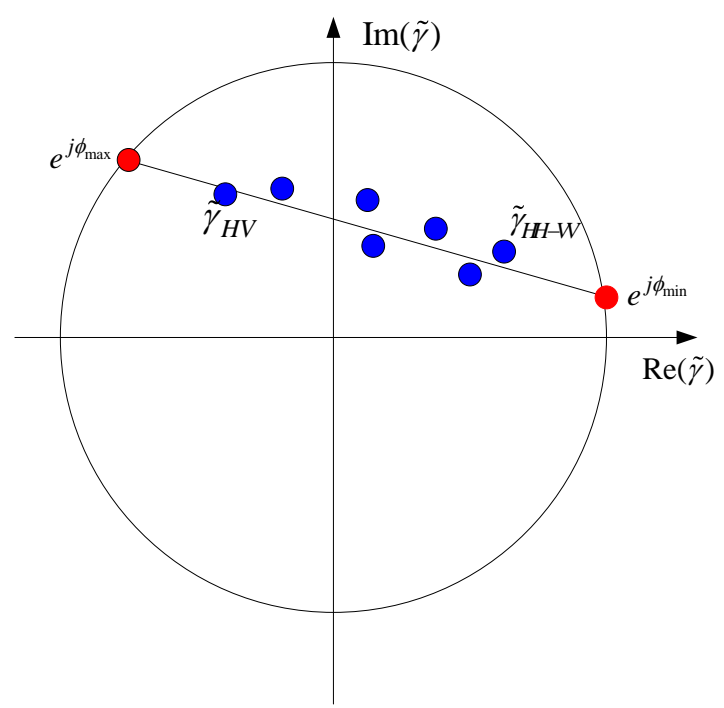

Figure 2. Total Least Squares Line Fit to Complex Coherence Data

\subsection{ESPIRT Algorithm}

The ESPRIT (Estimation of $\underline{\text { Signal }} \underline{\text { Parameters via }}$ Rotational Invariance $\underline{\text { Techniques) }}$ method is a high resolution method used to detect a signal inside noisy observations [12]. With SAR data, backscatter waves result from the sum of different scattering mechanisms. The interferometry phase depends strongly on the medium under observation. The signals acquired during a polarimetric interferometric measurement can be written as [2, 4]:

$$
\begin{aligned}
& S_{1}^{k l}=\sum_{m=1}^{d} \sigma_{m} \xi_{m}^{k l} e^{j \frac{4 \pi}{\lambda} R}+n_{1}^{k l} \\
& S_{2}^{k l}=\sum_{m=1}^{d} \sigma_{m} \xi_{m}^{k l} e^{j \frac{4 \pi}{\lambda}\left(R+\Delta R_{m}\right)}+n_{2}^{k l}
\end{aligned}
$$

where $k, l$ denote polarization combinations. The coefficient $\xi_{m}^{k l}$ is the polarization state of the $m$-th local scatterer in $\mathrm{kl}$ polarization channel. Each polarization state is assumed to be 
normalized with respect to the power of the local scatterer. $\sigma_{m}$ denotes the complex amplitude of the $m$-th scattered wave. Two factors $n_{1}^{k l}$ and $n_{2}^{k l}$ indicate the additive noise in $k l$ polarization channel of two SAR system, respectively.

Using matrix-vector expressions, the Eq. (10) can be rewritten as follows

$$
\begin{aligned}
& S_{1}=\left[S_{h h}^{1}, S_{h v}^{1}, S_{v h}^{1}, S_{v v}^{1}\right]^{T}=S \sigma+n_{1} \\
& S_{2}=\left[S_{h h}^{2}, S_{h v}^{2}, S_{v h}^{2}, S_{v v}^{2}\right]^{T}=S D \sigma+n_{2}
\end{aligned}
$$

In the case of backscattering in a reciprocal medium, the Eq. (11) can be represented by

$$
\begin{aligned}
& S_{1}=\left[S_{h h}^{1}, \sqrt{2} S_{h v}^{1}, S_{v v}^{1}\right]^{T}=S \sigma+n_{1} \\
& S_{2}=\left[S_{h h}^{2}, \sqrt{2} S_{h v}^{2}, S_{v v}^{2}\right]^{T}=S D \sigma+n_{2}
\end{aligned}
$$

The columns of the $3 \times 2$ matrix $S$ contain the polarization state of each local scatterer. In the ESPRIT algorithm, we define the overall data vector $\vec{x}$ and its correlation matrix $R_{x x}$ as

$$
R_{x x}=\left\langle\vec{x} \cdot \vec{x}^{* T}\right\rangle \text { and } \vec{x}=\left[S_{1}^{T}, S_{2}^{T}\right]^{T}
$$

where $\langle\bullet\rangle$ denotes the ensemble average in the data processing and $*$ represents the complex conjugation. The number of dominant scatterers can be estimated by the normalized eigenvalue $\left(\bar{\lambda}_{i}\right)$ of $R_{x x}$, which can be expressed by:

$$
\bar{\lambda}_{1} \geq \bar{\lambda}_{2} \geq \cdots \geq \bar{\lambda}_{d} \geq \bar{\lambda}_{d+1}=\bar{\lambda}_{6} \quad \text { and } \quad \bar{\lambda}_{j}=\frac{\bar{\lambda}_{j}}{\sum_{j=1}^{d} \bar{\lambda}_{j}}
$$

where $\bar{\lambda}_{j}$ is the eigenvalue of the matrix $R_{x x}$. When there exits only one dominant scatterer corresponding to maximum eigenvalues $(j=1)$ appears.

Using the eigenvectors corresponding to the dominant eigenvalues, the matrix $D$ can be estimated. Relation between the eigenvectors $\left(e_{1}, e_{2}, \cdots, e_{d}\right)$ and the matrices $S, D$ can be rewritten by

$$
\left[e_{1}, e_{2}, \cdots, e_{d}\right]=\left[\begin{array}{l}
F_{1} \\
F_{2}
\end{array}\right]=\left[\begin{array}{c}
S C \\
S D C
\end{array}\right]
$$

where $C$ is a $d \times d$ non-singular matrix. From Eq. (15) we can be derived the following equation

$$
F_{1} D F_{1}^{* T}=F_{2} F_{1}^{* T}
$$


This equation show that the diagonal elements of matrix $\mathrm{D}$ can be obtained by the complex eigenvalues of $F_{2} F_{1}{ }^{*} T$. Therefore, the interferometric phase of the $m$-th scatterer can be estimated by

$$
\phi_{m}=\arg \left(\lambda_{m}^{\prime}\right)
$$

Thus, when the matrix $D$ is estimated, polarization state of each local scatterer can also be estimated. The ESPRIT algorithm is summarized in Figure 3.
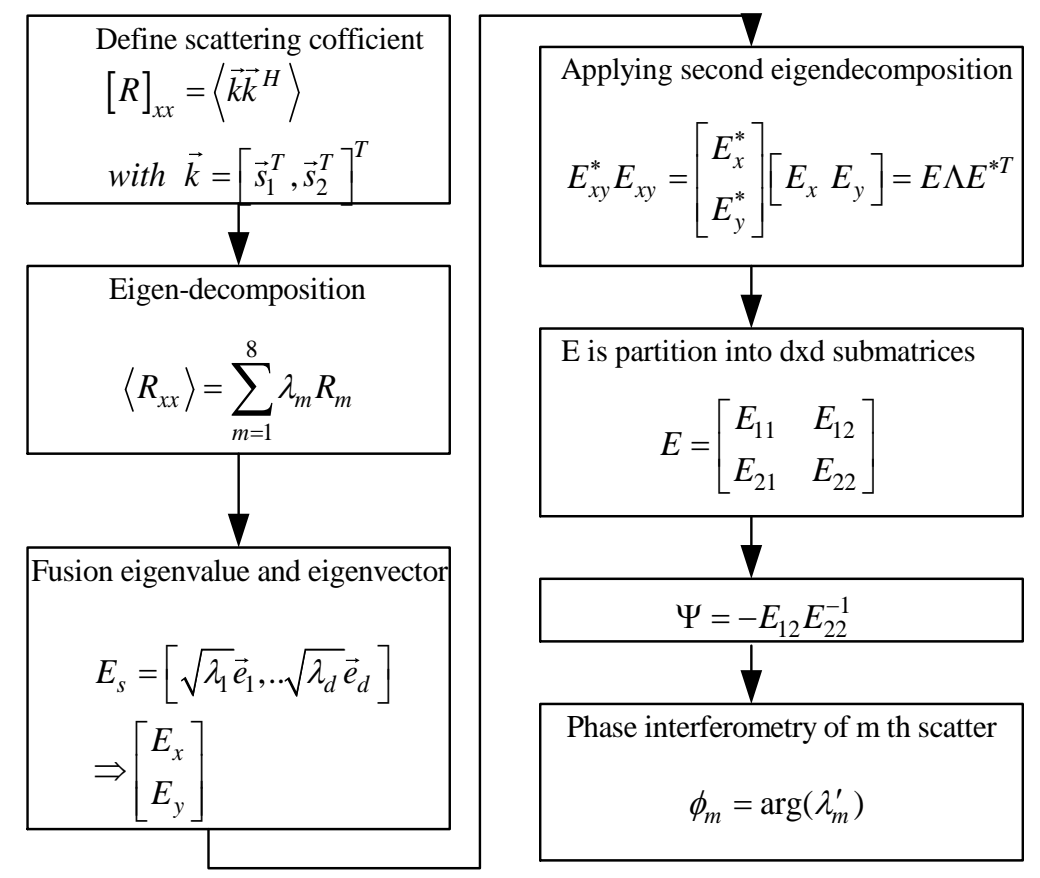

Figure 3. Diagram of ESPRIT Algorithm

\section{Forest Height Estimation using Hybrid Method with Compensate}

\subsection{A Hybrid Method Extracting Forest Height}

One of the simplest approaches to height estimation is to use the phase difference between interferogram as a direct estimate of height [10]. In this paper, height estimation can be extracted by hybrid total least square line fit method and ESPRIT algorithm.

In order to remove the phase bias and improve the accuracy of surface topography estimation, we employ a least square line fit to the multiple polarization channels to estimate the surface phase [11]. In this way we can avoid problems of any selected pair of points becoming too close, and thus minimize errors in the surface topography estimation.

The canopy phase can be estimated by using ESPRIT algorithm for dual polarization data set approximately. In this case, the measurement data vector in Eq. (12) may be re-defined as:

$$
\begin{aligned}
& S_{1}=\left[S_{h h}^{1}, \sqrt{2} S_{h v}^{1}, 0\right]^{T} \\
& S_{2}=\left[S_{h h}^{2}, \sqrt{2} S_{h v}^{2}, 0\right]^{T}
\end{aligned}
$$


The last element of Eq. (18) is a dummy component to create a null space of the signal subspace for the TLS-ESPRIT analysis. Finally, we choose eigenvalues maximum correspond to vegetation canopy phase to employ into height estimation. Hence, the forest height by phase differencing can be expressed as:

$$
h_{v}=\frac{\phi_{v}-\phi_{0}}{k_{z}}=(\Delta \phi) \frac{\lambda}{4 \pi} \frac{R \sin (\theta)}{B \cos (\theta-\delta)}
$$

where $\theta$ is the mean angle of incidence, $R$ the distance between radar and an observed point, $\delta$ the baseline tilt angle, $B$ the baseline and $\lambda$ the wavelength. The two phase $\phi_{v}$ and $\phi_{0}$ are the canopy phase and the underlying ground topography phase, respectively.

\subsection{Height Estimation from Coherence Amplitude Only}

In the case of the forest model the propagation through the volume is independent of polarization. Consequently, in Eq. (1) only the factor $m(\vec{\omega})$ is a function of polarization. This arises since the volume only complex coherence $\tilde{\gamma}_{v}$ becomes independent of polarization.

$$
\tilde{\gamma}_{v}\left(h_{v}, \sigma_{e}\right)=\frac{\int_{0}^{h_{v}} e^{\frac{2 \sigma_{e} z^{\prime}}{\cos (\theta)}} e^{j k_{z} z^{\prime}} d z^{\prime}}{\int_{0}^{h_{v}} e^{\frac{2 \sigma_{e} z^{\prime}}{\cos (\theta)}} d z^{\prime}}
$$

where $k_{z}$ is the vertical wavenumber and $\sigma_{e}$ denotes the extinction coefficient of electromagnetic wave, respectively.

Cloude proposed three-stage inversion method [5] for vegetable parameter estimation in 2003. In the limit, assuming the extinction approaches zeros $\sigma_{e} \rightarrow 0$, the volume only complex coherence $\tilde{\gamma}_{v}\left(h_{v}, \sigma_{e}\right)$ is the function which can only contain the height. Then the volume complex coherence $\tilde{\gamma}_{v}\left(h_{v}\right)$ is expressed by:

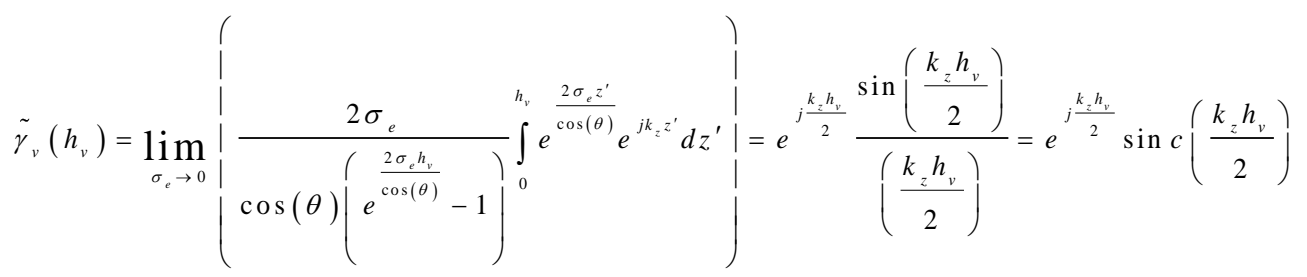

Since $\tilde{\gamma}_{v}\left(h_{v}\right)$ is given by a complex SINC function. The Eq. (21) show that two important features about $\tilde{\gamma}_{v}\left(h_{v}\right)$. Firstly, it shows that volume scattering provides an interferometric phase by half the real height. Secondly the SINC model is that the coherence amplitude falls with increasing height and hence phase variance increases with $h_{v}$. Therefore, we can use an estimate of measure coherence amplitude to estimate height (with a known baseline). The 
volume only coherence complex $\tilde{\gamma}_{v}$ can be replace with $\tilde{\gamma}_{v}\left(\vec{\omega}_{v}\right)$ so the forest height estimation from coherence amplitude only can be written as:

$$
h_{v}^{\text {co-amplitude }}=\frac{2 \arcsin c\left(\left|\tilde{\gamma}_{v}\left(\vec{\omega}_{v}\right)\right|\right)}{k_{z}}
$$

In particular, for short baselines we can expand $h_{v}^{\text {co-amplitude }}$ function as:

$$
h_{v}^{\text {co-amplitude }} \approx \frac{\pi-2 \arcsin \left(\left|\tilde{\gamma}_{v}\left(\vec{\omega}_{v}\right)\right|^{0.8}\right)}{k_{z}}
$$

The forest height estimation from coherence amplitude only in Eq. (23) is sensitive to variations in vertical structure of forest and is generally applied to compensate the underestimated height.

\subsection{Forest Height Estimation from L-band Single-Baseline PolInSAR Data using the Proposed Method}

In order to improve an accurate vegetation height estimate we first use the height that is estimated by hybrid total least squares line fit method and ESPRIT algorithm. However, phase centers of canopy layer, according to RVoG model, can lie anywhere between halfway and the top of the layer, and hence in general underestimates the true height. To progress, this error can be at least partly compensated by employing a coherence amplitude correction term. Finally, by combining these two terms with a scaling parameter $\eta$, we then obtain an accurate height algorithm that can compensate variation in structure, as shown in Eq. (24):

$$
\left.h_{v}=(\Delta \phi) \frac{\lambda}{4 \pi} \frac{R \sin (\theta)}{B \cos (\theta-\delta)}\right)+\eta \frac{\pi-2 \arcsin \left(\left|\tilde{\gamma}_{v}\left(\vec{\omega}_{v}\right)\right|^{0.8}\right)}{k_{z}}
$$

The first term in Eq. (24) represents the phase component while the second term is the coherence amplitude correction is weighted by $\eta$. It is selected to enhance accuracy for height estimation. If the medium has a uniform structure function then the phase centers locates in half-way of the layer, the weight is $\eta=0.5$. At the another extreme, if the structure function in the volume channel is localized near the top of the layer, then the phase height will be the true height, and the second term in Eq. (24) will approach zero. For that reason, the weight set as $\eta=0$. In order to reduce the error from the change of extinction coefficient and the vertical structure, we select $\eta=0.4$ [11]. The proposed method is summarized in Figure 4. 


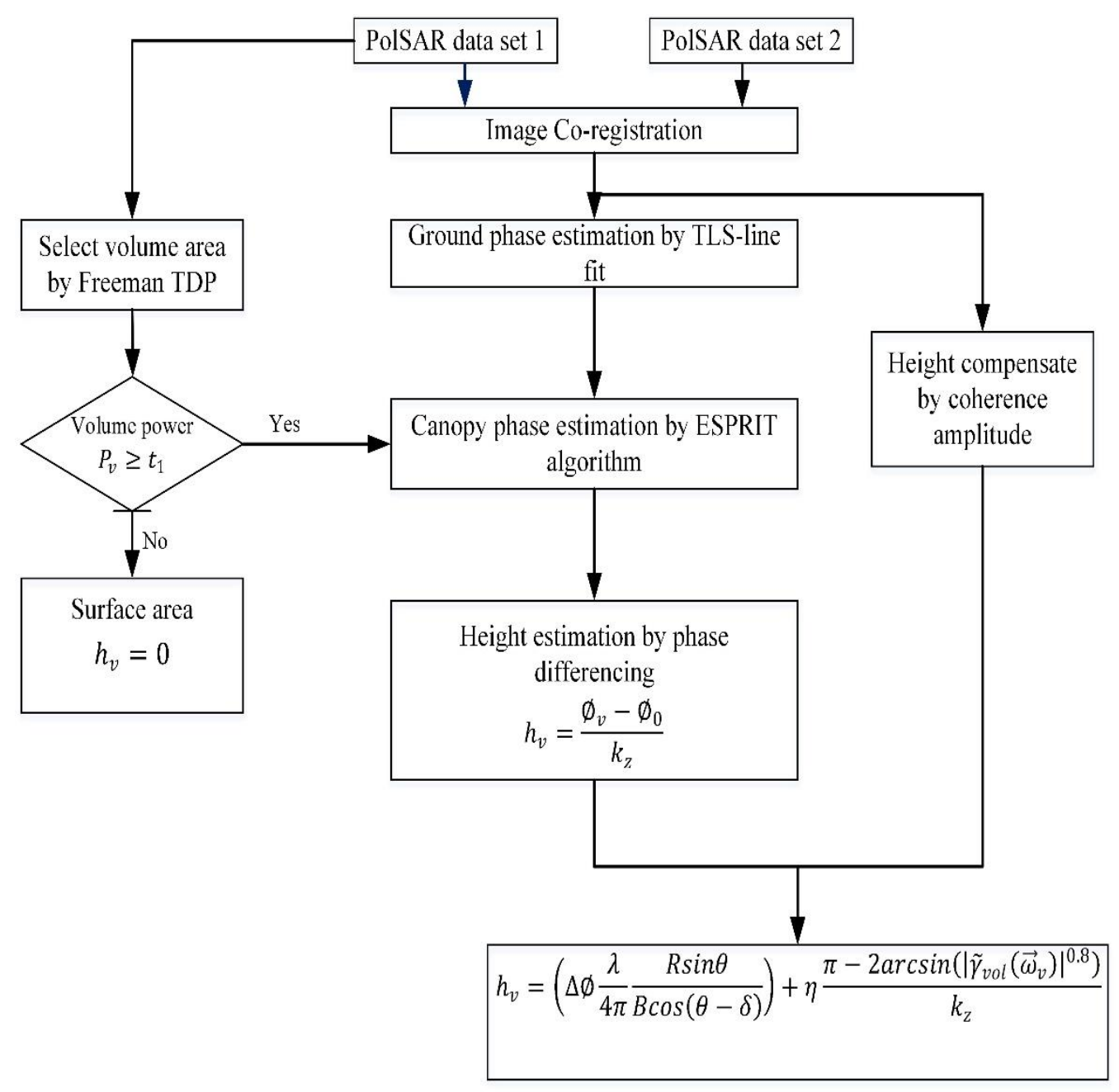

Figure 4. The Flow Chart of Proposed Method

\section{Experimental Results and Discussion}

The effective evaluation of the proposed approach is addressed but primarily in terms of the retrieved forest height estimation and ground phase. For such a purpose, we have applied the proposed method to a data set acquired from PolSARProSim software by Mark L. William [13], as well as spaceborne data acquired by ALOS PALSAR system from Japan Aerospace Exploration Agency (JAXA).

\subsection{Simulated PolInSAR data}

The proposed algorithm has been first test a simulated RVoG scenario, named as PINE in the PolSARProSim software, at $1.3 \mathrm{GHz}$ and at 30 degree angle of incidence considering different soil conditions and averaging window sizes. The interferometer is operated at $10.0 \mathrm{~m}$ horizontal and $1.0 \mathrm{~m}$ vertical baseline. The stand height $15 \mathrm{~m}$, and it is located on a $0.1 \%$ ground azimuth and $0.2 \%$ ground range slope. The forest stand occupies a $0.72854 \mathrm{Ha}$ area and stand density is $1000 \mathrm{stem} / \mathrm{Ha}$. Azimuth and slant range resolution are $1.0 \mathrm{~m}$ and $1.5 \mathrm{~m}$, respectively. 
Figure 5 shows the simulated scenario that it is the coniferous forest zone. Figure 6 (a) shows a red, green, blue (RGB) coding Pauli image of the forest scenario considered and the red line indicates the transect analyzed in this section, with 143 pixels in range and 131 pixels in azimuth. The top of image corresponds to far range, which can be identified due to the shadowing effect at the borders of the forest. The forest scenario considered is the placed above a Bragg surface with slightly sloped terrain. Figure 6 (b) shows the results of Freeman and Durden three component decomposition. In this figure, we show that the volume, double bounce and single bounce scattering represent corresponding with RGB coding color diagram: red channel (R), green channel $(\mathrm{G})$ and blue channel (B), respectively.

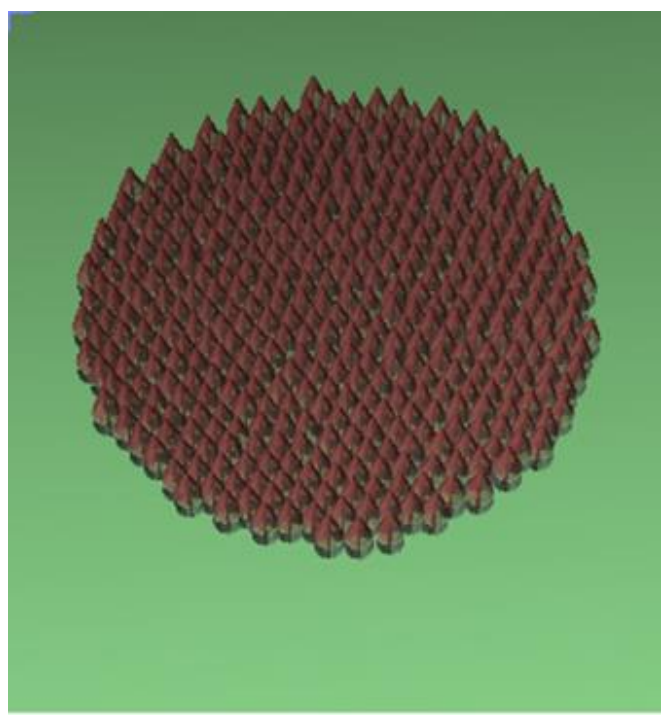

Figure 5. Scene of Simulation

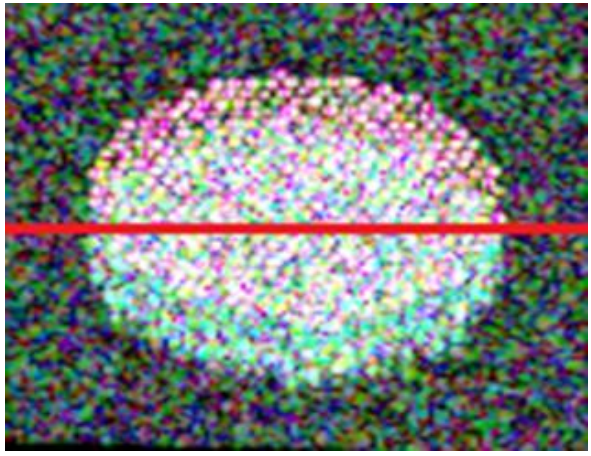

(a)

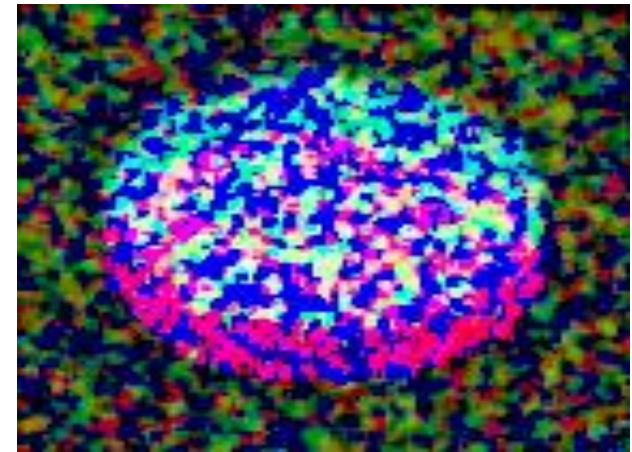

(b)

Figure 6. (a) Pauli Image on RGB Coding of Simulated Data, (b) Freeman and Durden Decomposition Result

Figure 7 is a plot of the forest height estimation of the proposed approach compared with the three-stage inversion method in the 126th row of azimuth transect line. Compared with the actual $15 \mathrm{~m}$ tree height, Table 1 indicates that the proposed approach is more accurate and has less error than the three-stage inversion method. The three-stage inversion process requires that the vertical structure and the temporal decorrelation is neglected and the 
minimum ground-to-volume scattering ratio needs to be lower than $-10 \mathrm{~dB}$ to secure around $10 \%$ accuracy [14]. The same with the three-stage inversion method, in order to improve the accuracy of ground phase estimation the proposed method also use multiple parameter least squares estimation. The ESPRIT technique can be detected local scattering centers corresponding to the canopy top and ground in the forest area but detection accuracy of the technique becomes worse for dense forest region due to the closer two phase centers [15]. In the proposed method, we implement Freeman and Durden decomposition technique to detect the volume scattering before applying the ESPRIT. So, the accuracy of canopy phase estimation is improved remarkable. Therefore, the forest height and extinction estimation by using three-stage inversion method is not reliable and only the underlying ground topography phase is reliable. Based on Figure 7 and Table 1 we can say that the forest height and ground phase estimation by using the proposed method is more accurate and reliable than its by using three-stage inversion method.

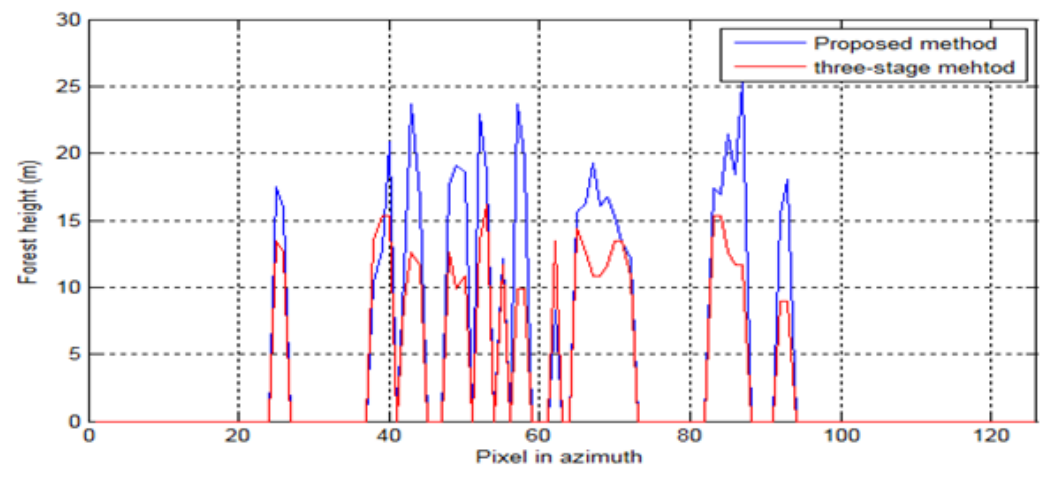

Figure 7. Plot of the Height Results Comparison

Table 1. Comparison between Three-Stage Inversion Method and Proposed Approach

\begin{tabular}{c|r|r|r|r|c}
\hline Parameter & $\begin{array}{r}\text { Average } \\
\text { height [m] }\end{array}$ & $\begin{array}{r}\text { Average } \\
\text { errors [m] }\end{array}$ & RMSE [m] & $\begin{array}{c}\text { Ground } \\
\text { phase [rad] }\end{array}$ & $\begin{array}{c}\text { Canopy phase } \\
{[\mathrm{rad}]}\end{array}$ \\
\hline Proposed & 15.0976 & -0.1764 & 5.6937 & -0.0142 & 2.9318 \\
\hline $\begin{array}{c}\text { Three-stage } \\
\text { inversion }\end{array}$ & 13.7840 & 1.2168 & 3.7418 & -0.0487 & - \\
\hline
\end{tabular}

The forest height estimation by using the proposed method is shown in Figure 8. In this figure it is shown that the peak differential of the forest height is located approximately at $15 \mathrm{~m}$ and most of forest height ranges from $12 \mathrm{~m}$ to $25 \mathrm{~m}$. The actual forest heights are quite well retrieve, except some pixels are overestimated but the almost of forest height in these pixels all less than $25 \mathrm{~m}$. The real effective tree height will be higher than these values so we can say that these results are acceptable. Likewise, the proposed method provides relative accuracy with small error, and is more accurate for vertical structural variations.

Figure 9 shows a three dimensional perspective view of the estimated height by the proposed approach for whole scenario. The mean forest height is $15.0976 \mathrm{~m}$ and the mean volume extinction coefficient is $0.2 \mathrm{~dB} / \mathrm{m}$. 


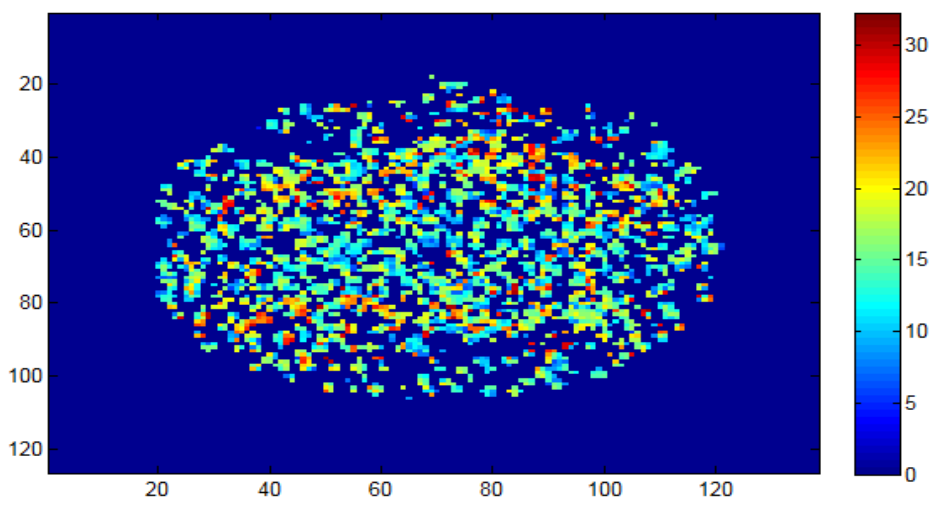

Figure 8. Forest Height Estimated by Proposed Approach

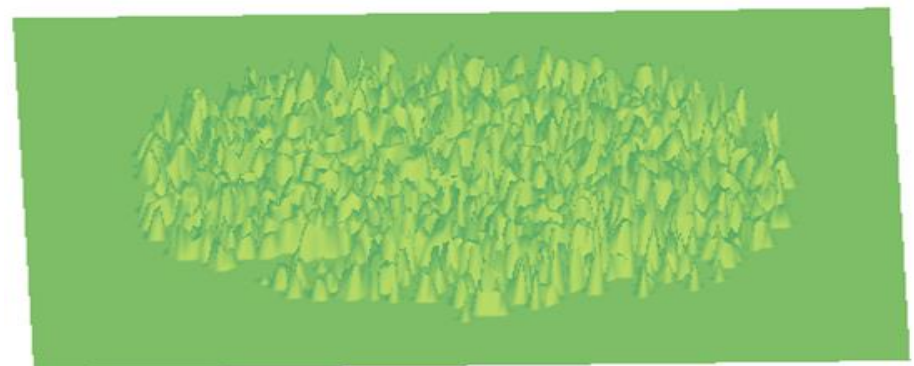

Figure 9. 3-D Perspective View of the Estimated Forest Height for Simulated Data

\subsection{Space Borne PolInSAR Data}

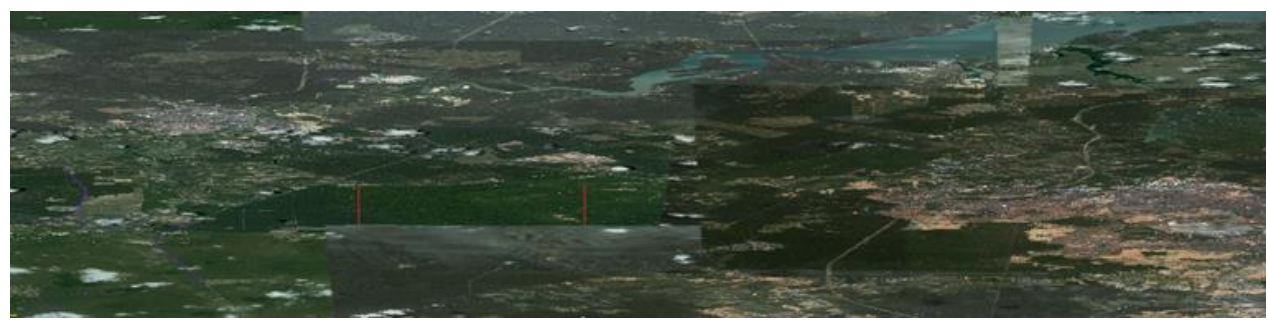

In this section, the effective evaluation of the proposed approach is also addressed but primarily in terms of the retrieved forest height. The data set using for testing proposed method is acquired from an image pair of Malaysia by the ALOS-PALSAR system, obtained on April 16th and June 1st, 2007, respectively. Its spatial resolution is $30 \mathrm{~m} \times 10 \mathrm{~m}$. They consist of quad-pol interferometric data at L-band with 21.5 degree angle of incidence and $877.5978 \mathrm{~m}$ baseline. The data set area, composed of $9217 \times 1248$, contain heterogeneous object such as forest areas, agricultural areas, river, lake, and urban area as shown in Fig. 10. As analyzed by Papathanassiou [19], the presence of temporal correlation coefficient leads to a decrease of the amplitude of the interferometric coherence, but do not affect the position of the effective phase centers. Furthermore, the amplitude of the interferometric coherence of test site data (after co-registration image and filtering procedures) almost greater than 0.6 and the forest height of proposed method is estimated by using the difference phase method. Hence, in this section, we neglect the effect of the temporal decorrelation. 
After co-registration of PolInSAR image, we select an evaluation area with 495 pixels in range and 327 in azimuth. The evaluation region has a mixed forestry, road and agricultural area. Figure 11 (a) is the optical image of the evaluation patch (b) HV amplitude image of test area for evaluation as forest and (c) is a composite image of the evaluation patch in the Pauli basis.

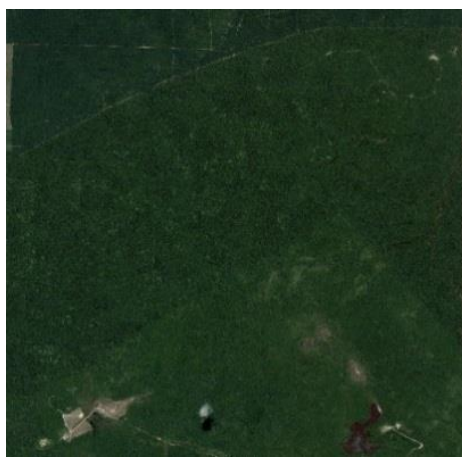

(a)

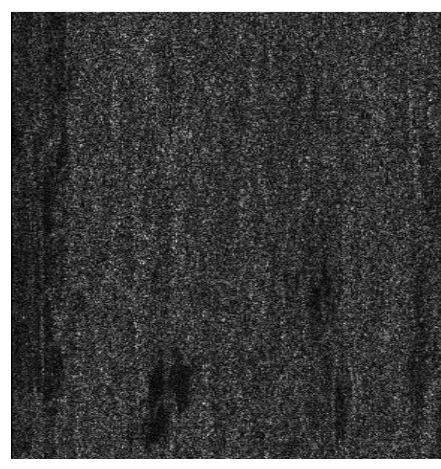

(b)

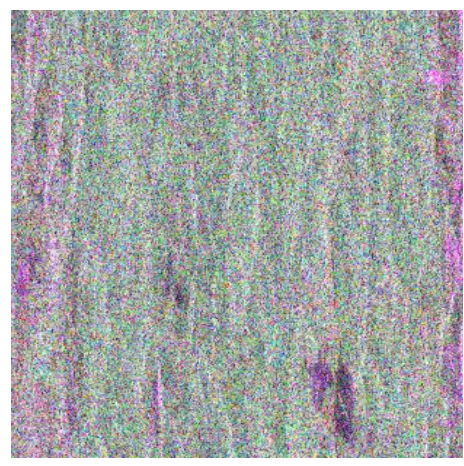

(c)

Figure 11. Data of Evaluation Patch, (a) Optical Image from Google Earth, (b) HV Amplitude Image, (c) Pauli Decomposition Image

The analysis has been performed on the azimuth transect. Along this transect, we can identify three types of scenes: red areas denote bare surface like agriculture and road, green areas represent forest.

Figure 12 plots the extracted forest height by the proposed approach in the azimuth line. This figure shows that the forest height estimation by proposed approach is located in a range from $15 \mathrm{~m}$ to $45 \mathrm{~m}$. Figure 13 shows the forest height estimation of proposed approach for the whole scene. The mean forest height is $26.4503 \mathrm{~m}$. In this figure it is shown that the forest height estimation at some pixels is overestimated but it is mostly less than $45 \mathrm{~m}$. The real effective tree height will be higher than these values so we can say that the results are acceptable.

Figure 14 shows a three dimensional perspective view of the estimated height by proposed approach for the whole scene. Table 2 shows the height estimation performance for the test site area.

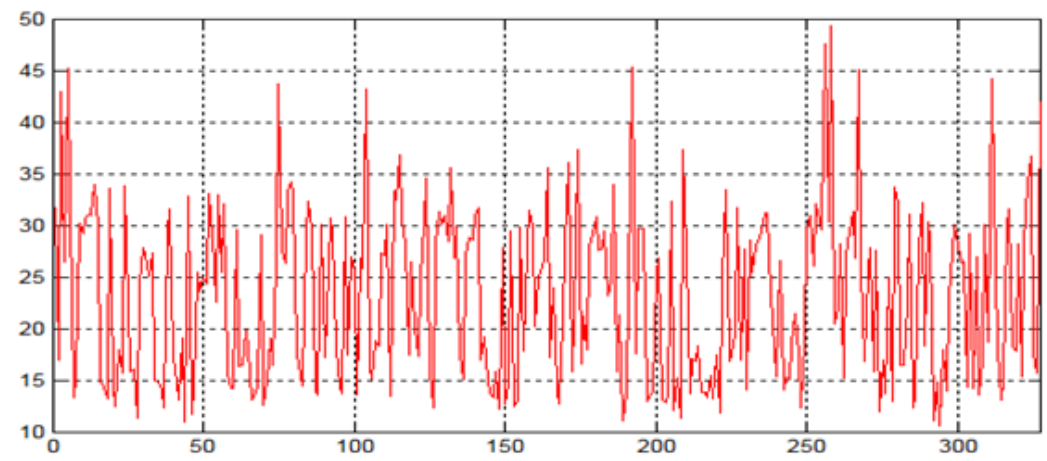

Figure 12. Extracted Forest Height of a Selected Row 


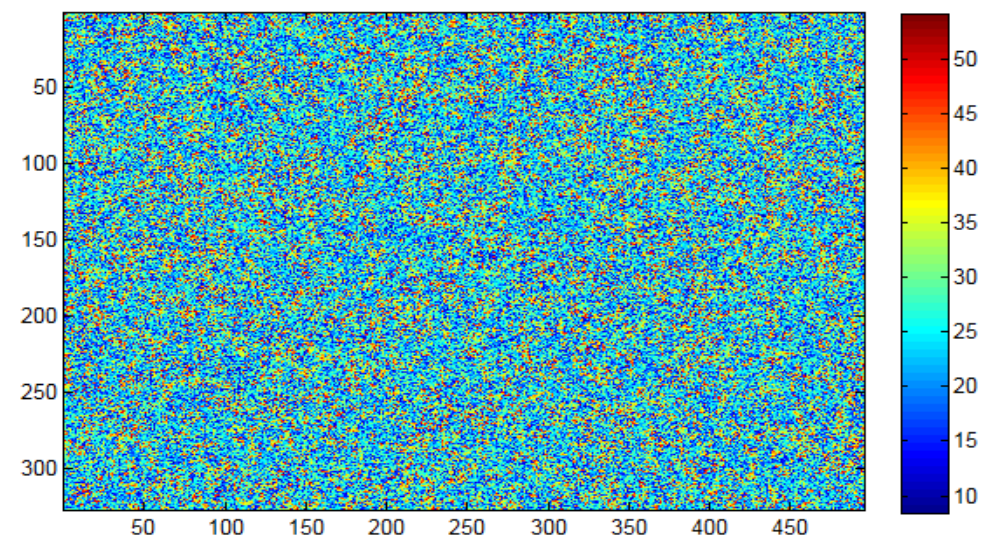

Figure 13. Forest Height is Estimated by Proposed Approach

Table 2. Forest Parameter Estimation for Selected Area

\begin{tabular}{ccccc}
\hline $\begin{array}{c}\text { Average } \\
\text { height }[\mathrm{m}]\end{array}$ & $\begin{array}{c}\text { Average } \\
\text { error }[\mathrm{m}]\end{array}$ & RMSE [m] & $\begin{array}{c}\text { Ground phase } \\
{[\mathrm{rad}]}\end{array}$ & $\begin{array}{c}\text { Canopy phase } \\
{[\mathrm{m}]}\end{array}$ \\
\hline 26.4503 & 1.7612 & 6.3983 & -0.0050 & 4.2305 \\
\hline
\end{tabular}

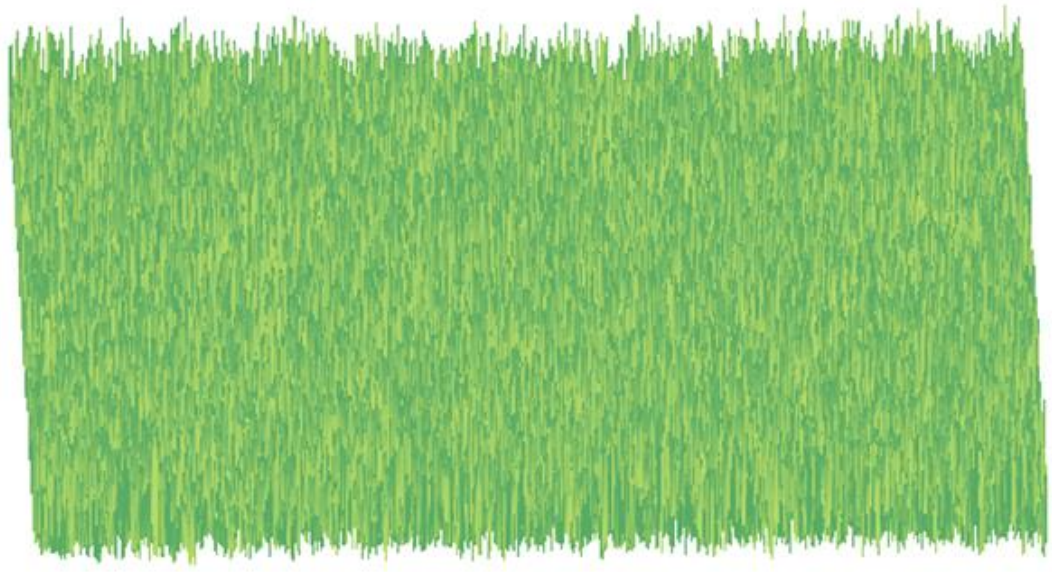

Figure 14. 3-D Perspective View of the Estimated Forest Height for the Evaluation Area

\section{Conclusion}

In this paper, in order to improve accuracy of height estimation we combined a hybrid method - total least square line fit, ESPRIT and coherence amplitude algorithm. Experimental result of the proposed approach versus the ESPRIT method have been presented. As demonstrated, the combination of these two approaches show that it is possible to retrieve forest height directly and accurately. Experimental results indicate that the forest parameters can be retrieved directly and more accurately by the proposed approach. In the future, further theoretical and experimental investigations will be done to improve the performance of the proposed approach. 


\section{Acknowledgements}

This work was supported by National S\&T Plans in Agriculture Area in the Twelfth FiveYear Period (No. 2011BAD08B02-03). This paper is a revised and expanded version of a paper entitled "Accuracy improvement method of forest height estimation for PolInSAR image", presented at IEEE/IET International Conference on Audio, Language and Image Processing, Shanghai, China, 2012 July 16-18.

\section{References}

[1] FAO, “Gobal forest resources assessment 2005”, FAO Forestry Paper, vol. 147, (2005).

[2] H. Yamada, Y. Yamaguchi, Y. Kim, E. Rodriguez and W. M. Boener, "Polarimetric SAR interferometry for forest analysis based on the ESPRIT algorithm", IEICE Transaction on Electron, vol E 84-C, no. 12, (2001), pp. 1917-2014.

[3] H. Yamada, M. Yamazaki and Y. Yamaguchi, "On scattering model decomposition of PolSAR and its application to ESPRIT-base Pol-InSAR", Proceeding of 6th European Conference on Synthetic Aperture Radar, Dresden, Germany, (2006) May 16-18.

[4] H. Yamada, M. Yamazaki and Y. Yamaguchi, "On scattering model decomposition of PolSAR and its application to ESPRIT-based PolInSAR", Proceeding of 6th European Conference on Synthetic Aperture Radar, (2006).

[5] S. R. Cloude and K. P. Papathanassiou, "Three-stage inversion process for polarimetric SAR interferometric", IEE Proceedings Radar, Sonar and Navigation, vol. 150, issue 3, (2003), pp. 125-134.

[6] T. Flynn, M. Tabb and R. Carande, "Direction estimation of vegetation parameters from covariance data in polarimetric SAR interferometry", Proceeding of IGARSS'02, (2002).

[7] T. M, C. R. Robust, "Inversion of vegetation structure parameters from low frequency polarimetric interferometric SAR", Proceeding of the IEEE International conference on Geoscience and remote sensing symposium, (2001).

[8] A. Freeman and S. L. Durden, "A three component scattering model to describle polarimetric SAR data", IEEE Transaction on Geoscience and Remote Sensing, vol. 36, no. 3, (1998), pp. 963-973.

[9] S. R. Cloude and K. P. Papathanassiou, "Polarimetric SAR interferometry", IEEE Transaction on Geoscience and Remote Sensing, vol. 36, no. 5, (1998), pp. 1551-1556.

[10] S. R. Cloude, "Pol-InSAR training course", Radio Science, (2005).

[11] S. R. Cloude, "Polarization Coherence Tomography", Radio Science, vol. 41, RS4017, (2006).

[12] R. Roy and T. Kailath, "ESPRIT-Estimation of Signal Parameter via Rotational Invariance Technique", IEEE Trans. Acoust, Speech, Signal Processing, vol. 37, (1989), pp. 984-995.

[13] M. L. Williams, "PolSARproSim: A coherent, Polarimetric SAR simulation of Forest for PolSARPro", http//earth.eo.esa.int/polsarpro/SimulatedDataSources.html, (2006).

[14] C. J. Hong, Z. Hong and W. Chao, "Comparision between ESPRIT algorithm and three-stage algorithm for PolInSAR", Proceeding of International conference on multimedia technology (ICMT), (2010), pp. 1-3.

[15] N. P. Minh, B. Zou and D. Lu, "Accuracy improvement method of forest height estimation for PolInSAR image", Proceeding of 11th International conference image processing, Beijing, China, (2012) October 2125, pp. 1830-1834.

[16] T. Mette, K. Papathanassiou and I. Hanjnsek, "Biomass estimation from polarimetric SAR interferometry over heterogeneous forest terrain", International Geoscience and Remote Sensing Symposium IGARSS, vol. 1, (2004), pp. 510-514.

[17] T. Mette, "Forest Biomass Estimation from Polarimetric SAR interferomtry", Ph.D dissertation, Techniche University Munchen, Germany, (2007).

[18] F. Garestier and T. Le Toan, "Forest modeling for forest height inversion using single baseline InSAR/PolInSAR data”, IEEE Transaction on Geoscience and Remote Sensing, vol. 48, no. 3, (2010), pp. 1528-1539.

[19] K. P. Papathanassiou and S. R. Cloude, "The effect of temporal decorrelation on the inversion of forest parameters from PolInSAR data", Proceeding of IEEE International conference on Geoscience and Remote Sensing Symposium, Toulouse, France, (2003) July 21-25, pp. 1429-1431. 


\section{Authors}

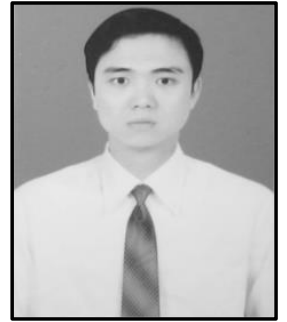

Nghia Pham Minh, Male, was born in 1980. He received the B.S., M.S. degrees from the Le Qui Don Technical University, Hanoi, Vietnam, in 2005 and 2008, respectively. Now he is pursuing Ph.D. degree in School of Electronics and Information Technology at Harbin Institute of Technology (HIT), Harbin, China. He currently focuses on synthetic aperture radar (SAR) image processing, polarimetric SAR, polarimetric SAR interferometry.

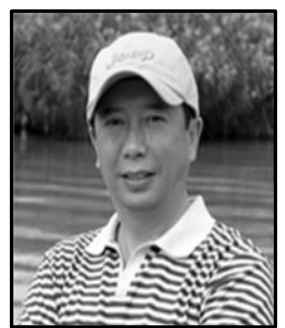

Chengyi Wang, he received BS degree from Northeast Forestry University, Harbin, China, in 1985, and the MS degree from Department of Chinese Academy of Forestry, Beijing, China, in 1999. Recently, he has published 40 papers and participated in 20 projects. He now undertakes "Twelfth Five Year" National Science and Technology Project in Rural Areas-Research and Demonstration of Ecological Restoration Technology in the Mining Area (No. 2011BAD08B02-03).

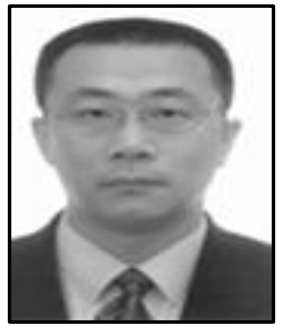

Bin Zou, Male, was born in 1968. He received the B.S. degree in electronic engineering from the Harbin Institute of Technology (HIT), Harbin, China, in 1990, the M.S. degree in space studies from the International Space University, Strasbourg, France, in 1998, and the Ph.D. degree in information and communication engineering from HIT, in 2001.

He was a Visiting Scholar in University of Manitoba, Winnipeg, Canada, from Oct. 2003 to Oct. 2004. He is currently a Professor and Vice Head of the Department of Information Engineering, School of Electronics and Information Technology, HIT. He currently focuses on synthetic aperture radar (SAR) image processing, polarimetric SAR, polarimetric SAR interferometry, hyper-spectral imaging and data processing.

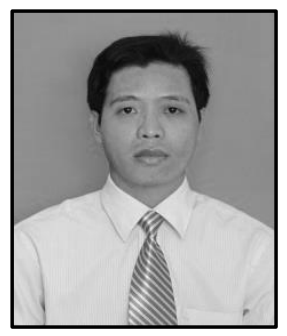

Quang Thi Nguyen, Male, was born in 1980. He received the B.S., M.S. degrees from the Le Quy Don Technical University, Hanoi, Vietnam, in 2004 and 2008, respectively. Now he is pursuing Ph.D. degree in School of Electronics and Information Engineering, Changchun University of Science and Technology. He currently focuses on blind deconvolution, image processing and pattern recognition.

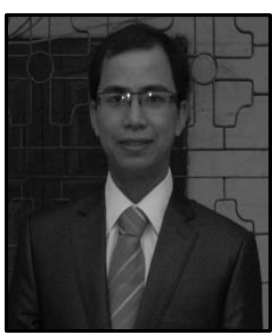

Van Nhu Le, Male, was born in 1982. He received the B.S degrees from the Le Quy Don Technical University, Hanoi, Vietnam, in 2007 and M.S dgree from Harbin Institute of Technology in 2012. Now he is pursuing Ph.D. degree in Research center for Space Optics Engineering, Harbin Institute of Technology, Harbin, China. He currently focuses image processing, optic image processing and design optic system. 
International Journal of Signal Processing, Image Processing and Pattern Recognition Vol.7, No.2 (2014) 